\title{
Transient behavior of a parametric amplifier with an added fourth-order interaction
}

\author{
C. Cabrillo, F. J. Bermejo, and P. Garcia-Fernandez \\ Instituto de Estructura de la Materia, Consejo Superior de Investigaciones Científicas, Serrano 123, 28006 Madrid, Spain \\ R. Toral, P. Colet, and M. San Miguel \\ Departamento de Fisica, Universitat de les Mles Balears, 07071 Palma de Mallorca, Spain
}

(Received 11 June 1991)

\begin{abstract}
The dynamical properties of an optical parametric amplifier with an added fourth-order (Kerreffect term) nonlinearity are studied by means of a computer simulation of a semiclassical nonlinear Langevin formulation. The transient statistics above threshold are analyzed in detail, and the relevance of the present results for the production of squeezed light in traveling-wave devices is finally discussed.

PACS number(s): 42.50.Dv, 42.65.Ky, 42.50.Md
\end{abstract}

\section{INTRODUCTION}

The possibility of achieving strong noise suppression in an optical parametric amplifier by means of an added Kerr-effect $\left(\chi^{(3)}\right)$ nonlinearity was pointed out by Tombesi [1] and further considered by Gerry and Rodrigues [2] some time ago. The rationale behind it was the fact that such a higher-order nonlinearity could well shorten the interaction time required to achieve the sought reduction in noise compared with the usual $\chi^{(2)}$ devices. An effective Hamiltonian was postulated comprising both the usual terms appearing in the description of a conventional optical parametric amplifier and an added fourth-order term which was found to enhance the squeezing properties of the model device at least in the initial stages of the evolution. A previous work [3] was devoted to the study of the stability of a more realistic model where noise sources arising from the coupling to a phonon bath were explicitly taken into account. The presence of the nonlinear contribution was found to be responsible for the appearance of a pitchfork bifurcation at threshold leading to bistable behavior above this point. In this paper the temporal evolution of such a model system is analyzed in detail. Because of the semiclassical nature of the treatment, which was carried out in a Wigner-representation frame, the transient behavior could be analyzed by means of a computer simulation of the coupled stochastic equations (provided that the analysis is not carried out in the vicinity of the instability point).

The squeezing characteristics both during the transient evolution and once the steady states above threshold have been reached have been studied in order to compare the noise reduction with those attainable by means of purely Kerr-effect interactions. In this latter respect, the interest was focused on the characterization of the statistics of the output field since it was shown previously $[4,5]$ that "amplitude-squeezed" states could be produced by systems which are describable by means of anharmonicoscillator Hamiltonians, such as those currently used to study the dynamics of fourth- and higher-order Kerr non- linearities.

The outline of the paper is as follows. The field statistics of the stationary states above threshold is considered first in Sec. II. The transient behavior as well as some considerations about the underlying assumptions are then examined in Secs. III and IV. Finally the main conclusions and the relevance of the present findings are commented in Sec. V.

\section{STUDY OF THE SQUEEZING FEATURES OF THE STATIONARY STATES ABOVE THRESHOLD}

In order to study the interaction of an intense laser beam of frequency $2 \Omega$-the pump beam-with the suitable nonlinear medium, one should be able to solve the IIeisenberg equations associated to the corresponding effective IIamiltonian. This is in general a difficult and still open problem. In this paper and following the work described in Ref. [3], we make use of a semiclassical aproach based on a Wigner (symmetric) phase-space representation [6]. This approach allows the representation of the evolution of the system in terms of two coupled real stochastic differential equations, provided that a certain linearization is valid. The resulting equations are then more convenient for computational purposes than their Heisenberg-Langevin counterparts. The details of the method are given in Ref. [3]. We give here only a brief outline of the main results.

The evolution of the annihilation operator is replaced by a complex stochastic process $\alpha=x(t)+i y(t)$. Removing the oscillatory motion of $\alpha(t), x(t)$ and $y(t)$ (the quadratures of the optical field) satisfy the following Langevin equations:

$$
\begin{aligned}
& \frac{d x}{d t}=(-\gamma+\kappa) x+\Gamma y\left(y^{2}+x^{2}\right)+L_{x}, \\
& \frac{d y}{d t}=(-\gamma-\kappa) y-\Gamma x\left(y^{2}+x^{2}\right)+L_{y},
\end{aligned}
$$

where $\gamma$ and $\kappa$ are the loss and gain parameters, respec- 
tively, and $\Gamma$ is the strength of the nonlinear (fourthorder) term of the Hamiltonian. Rewriting the above equations in terms of the dimensionless time $\tau=(\gamma+\kappa) t$, we get

$$
\begin{aligned}
& \frac{d x}{d \tau}=\mu x+\nu y\left(y^{2}+x^{2}\right)+\tilde{L}_{x}, \\
& \frac{d y}{d \tau}=-y-\nu x\left(y^{2}+x^{2}\right)+\tilde{L}_{y},
\end{aligned}
$$

where we have introduced the dimensionless constants

$$
\mu=\frac{\kappa-\gamma}{\kappa+\gamma}, \quad \nu=\frac{\Gamma}{\gamma+\kappa}
$$

and $\tilde{L}_{x}$ and $\tilde{L}_{y}$ are real Gaussian white noises of zero mean and correlations given by

$$
<\tilde{L}_{i}(t) \tilde{L}_{j}(t)>=\bar{D} \delta_{i j} \delta\left(t-t^{\prime}\right)
$$

where $\bar{D}$ is defined by

$$
2 \bar{D}=\frac{\gamma}{\gamma+\kappa}(1+2 \bar{n})
$$

and $\bar{n}$ depends on the temperature of the reservoir. The semiclassical equations (2) are the basis of our subsequent analysis.

A degenerate parametric amplifier operating above threshold gives rise (when pump depletion is accounted for) to two stationary states symmetrically located with respect to the origin $[7,8]$. In a Wigner-representation frame, the fluctuations can be graphically represented in the quadrature phase space by means of an error ellipse so that the quadrature noise components are just the projections of the ellipse contour on the two axes $[9,10]$. In this case, the orientation of the error ellipse is such that its main (larger) principal axis lies along the $x$ axis (i.e., the phase-sensitive noise amplification makes this quadrature the most amplified one and the converse is true for the other component). Such particular redistribution of the noise is a general feature of an optical parametric amplifier and does not depend upon the relationship between pump and loss.

It is worth noticing that the bistable behavior of our model system arises from the fourth-order interaction term, and not from the pump depletion (not considered in this model). The only feature that remains in our case is the symmetry with respect to the origin. In fact, Eqs. (2) give rise above threshold (i.e., $\mu>0$ and in addition to the trivial solution $x=y=0$ ) to two stationary states symmetrically located with respect to the origin. Namely, the stationary states $\left(x_{s}, y_{s}\right)$ are given by

$$
x_{s}= \pm \frac{\mu^{1 / 4}}{\sqrt{\nu(1+\mu)}}, \quad y_{s}=-\sqrt{\mu} x_{s}
$$

As a result, the stationary states are not in the $x$ axis and the principal axes of the error ellipses are not parallel to the coordinate axes. It is interesting, then, to study the orientation of the error ellipse seeking special directions of the ellipse main axis, i.e., perpendicular to the

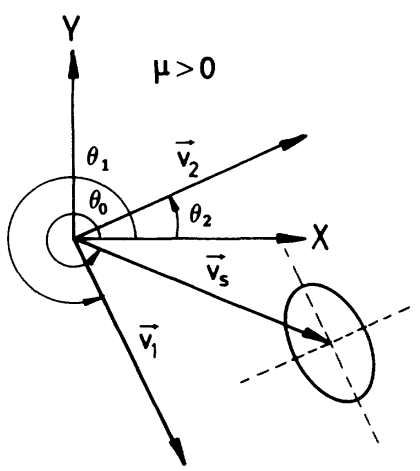

FIG. 1. The different angles and vectors involved in the problem. The coordinates $x$ and $y$ are the quadratures of the electric field and the ellipse represents the error contour in a symmetric phase-space representation of a steady state above threshold.

position vector of the stationary state (pure "amplitude squeezing") or parallel to it (pure "phase squeezing").

The error ellipse in the stationary states is defined from the variance matrix $\sigma\left(\sigma_{x} \equiv \sigma_{11}=\left\langle x^{2}>-<x\right\rangle^{2}\right.$, $\sigma_{x y} \equiv \sigma_{12}=\sigma_{21}=<x y>-<x><y>, \sigma_{y} \equiv \sigma_{22}$ $=<y^{2}>-<y>^{2}$ ). This is obtained by allowing small fluctuations $\delta x$ and $\delta y$ around the steady states and considering the linearized version of the stochastic equations (2)

$$
\left(\begin{array}{l}
\delta \dot{x} \\
\delta \dot{y}
\end{array}\right)=A\left(\begin{array}{l}
\delta x \\
\delta y
\end{array}\right)+\left(\begin{array}{c}
\tilde{L}_{x} \\
\tilde{L}_{y},
\end{array}\right)
$$

where $A$ is the drift matrix. The variance matrix $\sigma$ is then the solution of the following equation [11]:

$$
A \sigma+\sigma A^{T}=-\bar{D} \underline{I} \text {. }
$$

As it was shown in Ref. [3], the drift matrix $A$ depends only on the $\mu$ parameter. So $\sigma$ only depends upon $\mu$ and $\bar{D}$. Taking $\tilde{\sigma}=\sigma \bar{D}^{-1}$ we have

$$
A \tilde{\sigma}+\tilde{\sigma} A^{T}=-\underline{I} \text {. }
$$

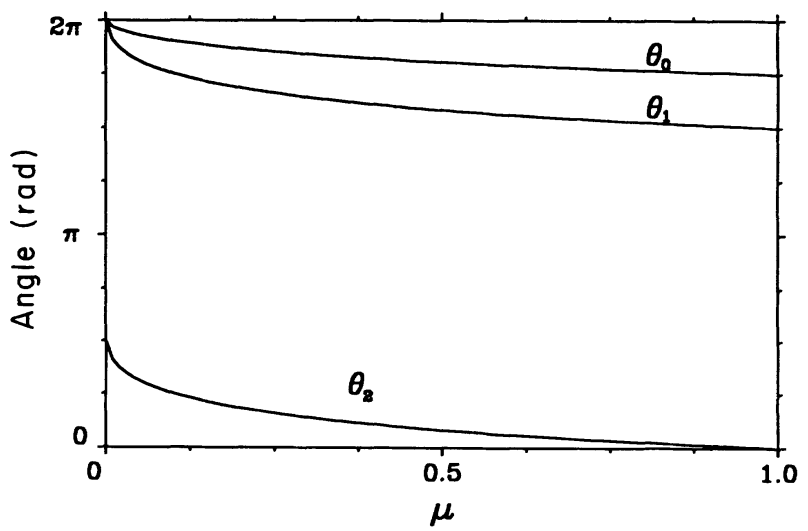

FIG. 2. The angles illustrated in Fig. 1 vs the parameter 


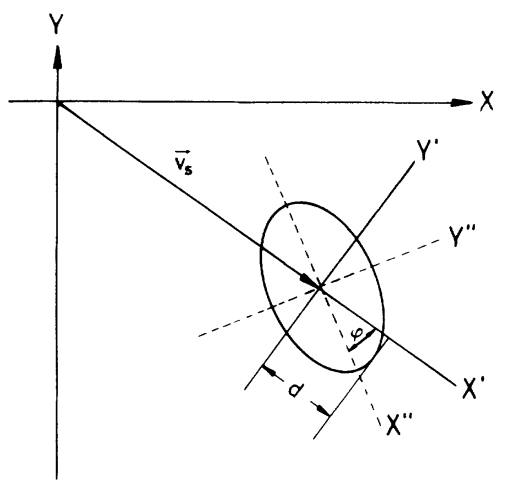

FIG. 3. Illustration of the projection of the error ellipse onto the position-vector direction.

Therefore $\tilde{\sigma}$ only depends on $\mu$ and has the same principal axes as $\sigma$ since $\bar{D}$ is a real number. The position vector of the stationary state $\mathbf{V}_{s}$ only depends on the $A$ matrix and, therefore, only on the parameter $\mu$.

Figure 1 illustrates the different angles involved in the problem. $\quad \mathbf{V}_{1}$ and $\mathbf{V}_{2}$ are eigenvectors of $\tilde{\sigma}$ in the maximum- and minimum-variance directions, respectively. The expressions for the different angles are

$$
\begin{aligned}
& \theta_{0}=\arctan \left(\frac{V_{s y}}{V_{s x}}\right), \\
& \theta_{1}=\arctan \left(\frac{V_{1 y}}{V_{1 x}}\right), \\
& \theta_{2}=\arctan \left(\frac{V_{2 y}}{V_{2 x}}\right),
\end{aligned}
$$

where the values for the angles are considered to be within the interval $[0,2 \pi]$. A numerical evaluation of this expression has been done. The representation of the different angles against $\mu$ is shown in Fig. 2. A $\mathbf{V}_{1}$ vector parallel or antiparallel to $\mathbf{V}_{s}$ would represent an intersection between the curves $\theta_{0}(\mu)$ or $\theta_{0}(\mu)-\pi$ with $\theta_{1}(\mu)$ and a $\mathbf{V}_{2}$ vector parallel or antiparallel to $\mathbf{V}_{s}$ would represent an intersection with $\theta_{2}(\mu)$. So, the model considered here does not show pure "phase" or "amplitude squeezing." However it is still possible that the projection of the error ellipse onto the $\mathbf{V}_{s}$ direction be less than $\frac{1}{2}$ which would represent a value smaller than that of a coherent

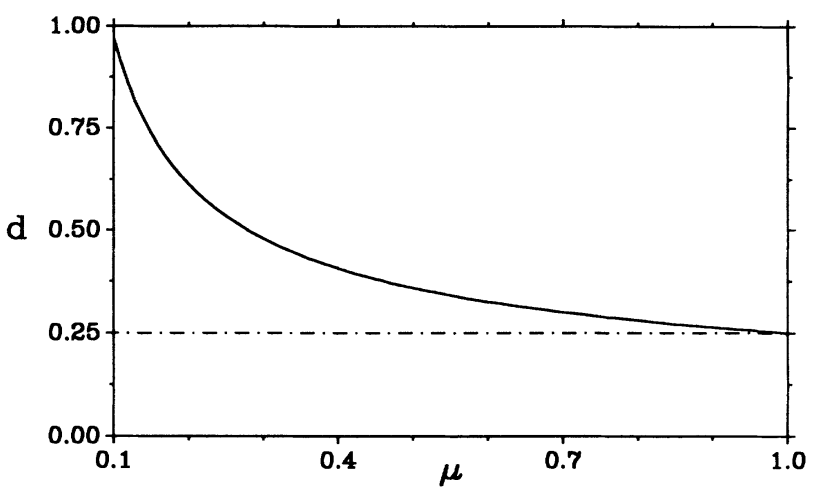

FIG. 4. The projection of the error ellipse onto the position-vector direction vs the parameter $\mu$.

state and, therefore, a state of sub-Poissonian light. The equation for the error ellipse in the $x^{\prime \prime}$ and $y^{\prime \prime}$ axes shown in Fig. 3 is

$$
\frac{\left(x^{\prime \prime}\right)^{2}}{\sigma_{11}^{2}}+\frac{\left(y^{\prime \prime}\right)^{2}}{\sigma_{22}^{2}}=1,
$$

where $\sigma_{11}$ and $\sigma_{22}$ are in this frame the maximum and minimum variances of the state. Transforming the $\left(x^{\prime \prime}, y^{\prime \prime}\right)$ axes to the $\left(x^{\prime}, y^{\prime}\right)$ axes by a rotation of angle $\varphi$ we obtain the equation in the $\left(x^{\prime}, y^{\prime}\right)$ frame with a general form

$$
a(\varphi)\left(y^{\prime}\right)^{2}+b(\varphi) x^{\prime} y^{\prime}+c(\varphi)\left(x^{\prime}\right)^{2}-1=0 .
$$

Taking (12) as a second-degree equation for $y^{\prime}$ the solution has the general form

$$
y^{\prime}=\frac{-b(\varphi) x^{\prime} \pm \sqrt{\Delta\left(\varphi, x^{\prime}\right)}}{2 a(\varphi)}
$$

where $\Delta\left(\varphi, x^{\prime}\right)$ is equal to $\left[b(\varphi) x^{\prime}\right]^{2}-4 a(\varphi)\left[c(\varphi)\left(x^{\prime}\right)^{2}-1\right]$. In order to obtain the $x^{\prime}$ values for which $y^{\prime}$ is single valued, the following condition must hold:

$$
\Delta\left(\varphi, x_{p}^{\prime}\right)=0
$$

In this way we obtain

$$
d=\left|x_{p}\right|=\left(\frac{\left(\frac{\cos \varphi}{\sigma_{22}}\right)^{2}+\left(\frac{\sin \varphi}{\sigma_{11}}\right)^{2}}{\left[\left(\frac{\cos \varphi}{\sigma_{22}}\right)^{2}+\left(\frac{\sin \varphi}{\sigma_{11}}\right)^{2}\right]\left[\left(\frac{\cos \varphi}{\sigma_{11}}\right)^{2}+\left(\frac{\sin \varphi}{\sigma_{22}}\right)^{2}\right]-\left(\frac{1}{\sigma_{22}^{2}}-\frac{1}{\sigma_{11}^{2}}\right)^{2} \cos ^{2} \varphi \sin ^{2} \varphi}\right)^{1 / 2} .
$$

Since $\varphi, \sigma_{11}$, and $\sigma_{22}$ only depend on $\mu, d$ must have the same dependence. Figure 4 shows a plot of $d$ versus $\mu$. It is clear that the produced states are not of sub-Poissonian character since $d(\mu)$ never goes below the 0.25 value.

\section{TRANSIENT BEHAVIOR}

The numerical simulation uses a stochastic first-order Euler scheme as explained in the Appendix of Ref. [12]. 
The Gaussian white noise has been generated by means of the Box-Muller-Wiener transformation [13] and an integration step of $10^{-3}$ has been used. The temporal dependence is given by the dimensionless time $\tau=(\gamma+\kappa) t$.

We have not considered thermal fluctuations $(\bar{n}=0)$ and $\gamma / \kappa$ (loss-pump ratio), $\Gamma / \kappa$ (fourth- to second-order strength ratio) as independent parameters in all calculations. The statistical averages were taken over 10000 trajectories when dealing with quadrature statistics and 20000 for photon-number statistics.

\section{A. Below threshold}

Figure 5 shows the temporal dependence of the quadrature variances ( $\sigma_{22}$ is the minimum quadrature variance and $\sigma_{11}$ is the maximum quadrature variance hereafter). For values of $\Gamma / \kappa$ within the range $[0.02,0.002]$ used in this work no influence of the nonlinear term has been found within our numerical precision. Therefore the transient behavior in this regime is essentially governed by the pure two-photon interaction.

\section{B. Above threshold}

As stated above, the nonlinear term included in our model system leads to the appearance of bistable behavior above threshold. In what follows we will briefly describe the transients.

Making use of the symmetry with respect to the origin of the evolution equations, averages over trajectories leading to the same final state were taken. As a consequence, the stationary variances calculated in this manner are the same as those reported in Ref. [3]. All the simulations presented here have 500 "measures" with a time step $\Delta \tau=0.15$.

It was clear from the outset that two different regimes could be defined depending on the value of the parameter $\gamma / \kappa$. While oscillations do not appear for large enough values of $\gamma / \kappa$, a rich oscillatory behavior was present when small enough values of the parameter $\gamma / \kappa$ were used. Since both regimes exhibit a rather distinctive phenomenology they are, in what follows, considered separately.

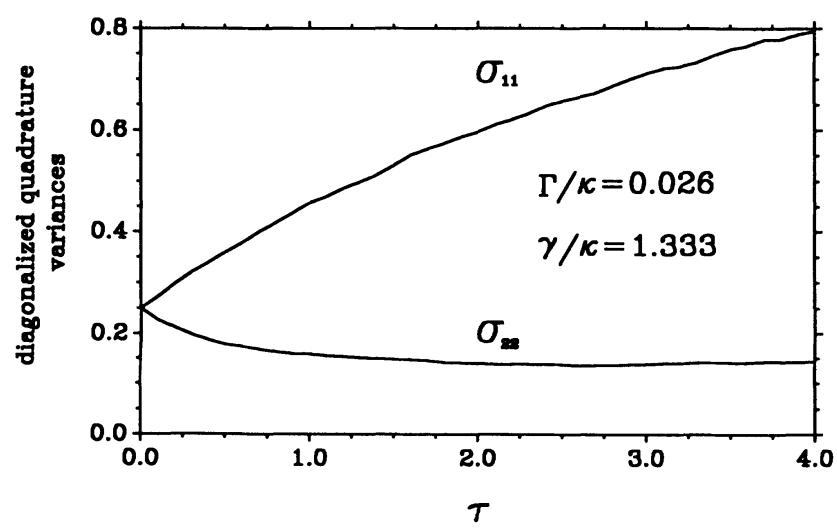

FIG. 5. Evolution of the maximum and minimum quadrature variances below threshold.

\section{Overdamped regime}

Figure 6 shows the evolution of the minimum variance for different values of the parameters. All the curves show an "oversqueezing" region [1,2] which corresponds to a pure two-photon interaction [no influence of the nonlinear term around the $(0,0)$ unstable point]. The value of $\sigma_{22}$ in this region coincides with the linearized theory around the unstable point [14,15] (second formula of Eq. (4.3) in Ref. [3]).

Figure $6(\mathrm{a})$ displays the influence of the $\Gamma / \kappa$ parameter. We have chosen $\gamma / \kappa=0.64$ to ensure that no jumps between stationary states occur [3]. A larger value of $\Gamma / \kappa$ involves an approach to the stationary states that implies a reduction of the two-photon interaction region and a quieter transient.

Figure 6(b) shows the dependence with $\gamma / \kappa$. A larger value of $\gamma / \kappa$ implies also an approach of the stationary states, but in this case the "oversqueezing" and stationary values of $\sigma_{22}$ get closer while $\gamma / \kappa$ increases, reaching the same value in the limit $\gamma / \kappa=1$. Therefore in this case an increase of $\gamma / \kappa$ implies a larger period of oversqueezing, a larger value of $\sigma_{22}$ in this region, and a quieter transient.

In Fig. 7 we show a transient with a coherent initial state other than the vacuum. This initial state is such that $|\alpha(0)| \Gamma /(\gamma+\kappa)=1$ is satisfied. In this way it reproduces the most favorable initial condition used by Tombesi [1]. The result shows how the squeezing is

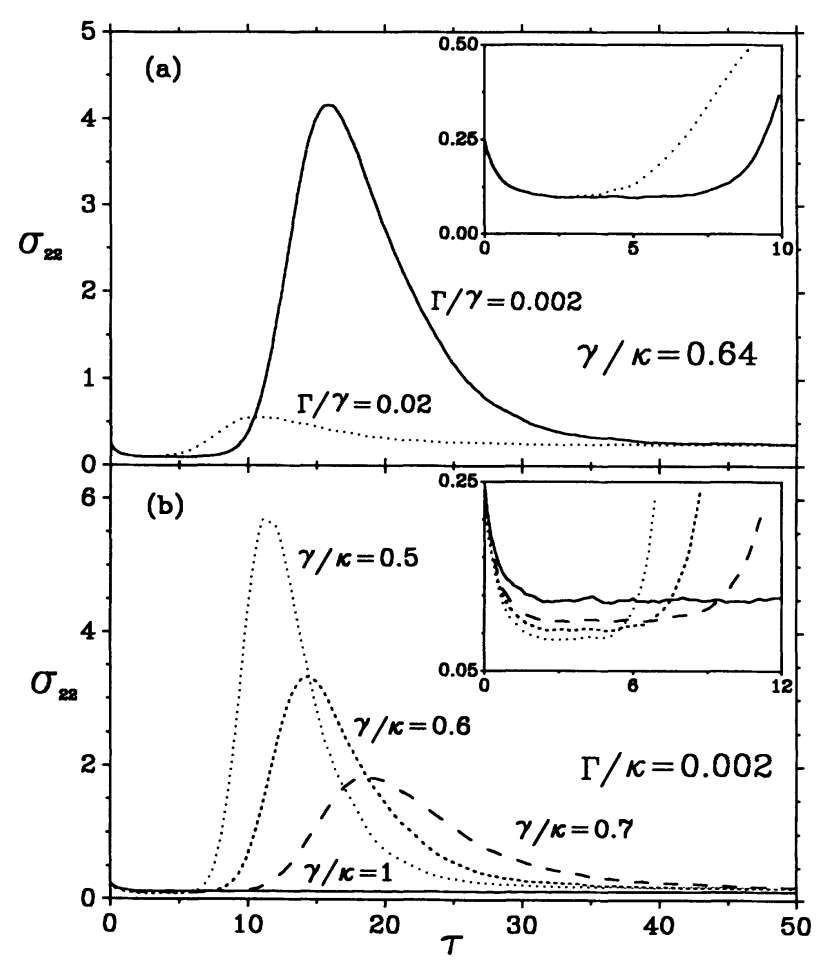

FIG. 6. Evolution of the minimum quadrature variance above threshold for a coherent-vacuum initial state. The dependence with respect to the parameter $\Gamma / \kappa$ is shown in (a) and with respect to the parameter $\gamma / \kappa$ in (b). The insets show the initial stages of the evolution. 


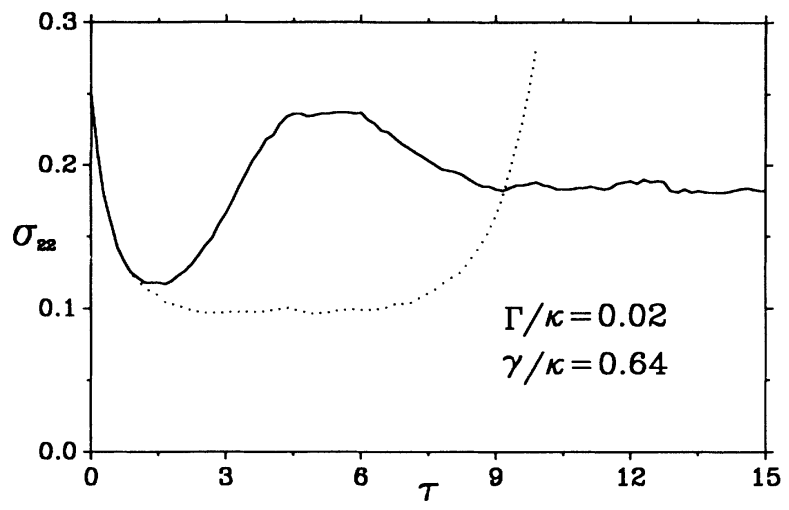

FIG. 7. Evolution of the minimum quadrature variance for a nonvacuum initial state compared with the same for a coherent-vacuum initial state. Solid line: coherent initial state such that $|\alpha(0)| \Gamma /(\gamma+\kappa)=1$. Dotted line: coherentvacuum initial state.

revoked before it reaches a value smaller than that of a pure two-photon interaction as Gerry and Rodrigues have shown for a lossless case [2].

\section{Oscillatory regime}

When $\gamma / \kappa$ tends to small enough values, damped oscillations appear on the different calculated variables. Besides the diagonalized variances for the quadratures, the average photon number and variance have been obtained, allowing a clearer vision of the oscillations. Considering that a Wigner (symmetric) representation in the phase space has been used and taking into account the commutation relations between quadrature operators we have

$$
\begin{aligned}
& <\hat{n}>=<\hat{a}^{\dagger} \hat{a}>=<\hat{x}^{2}+i[\hat{x}, \hat{y}]+\hat{y}^{2}> \\
& =\left(x^{2}\right)_{\mathrm{cl}}+\left(y^{2}\right)_{\mathrm{cl}}-\frac{1}{2} \\
& <(\Delta \hat{n})^{2}>=\left(x^{4}\right)_{\mathrm{cl}}+\left(y^{4}\right)_{\mathrm{cl}}-\left[\left(x^{2}\right)_{\mathrm{cl}}+\left(y^{2}\right)_{\mathrm{cl}}\right]^{2} \\
& +2\left(x^{2} y^{2}\right)_{\mathrm{cl}}-\frac{1}{4},
\end{aligned}
$$

where $\left\langle>\right.$ and ()$_{\mathrm{cl}}$ indicate quantum and classical averages, respectively.

The evolution of the mean photon number and variance is shown in Fig. 8. Two cases, one in the overdamped regime and one in the oscillatory regime, are displayed. Figure 9 shows the diagonalized variances for the quadratures for the coherent vacuum as initial state.

The oscillations in the quadrature quantities show an interesting double structure which is not present in the standard parametric amplifier [15]. A closer look at a display of several trajectories (Fig. 10) reveals a large temporal dispersion in the starting times (the times in which each trajectory reaches an observable value of the photon number). This temporal dispersion seems to be constant, i.e., each trajectory seems approximately to be just a shifted replica of one of them. A passage-time (PT) statistic has been computed for twelve different photonnumber reference values scanning the first peak in the

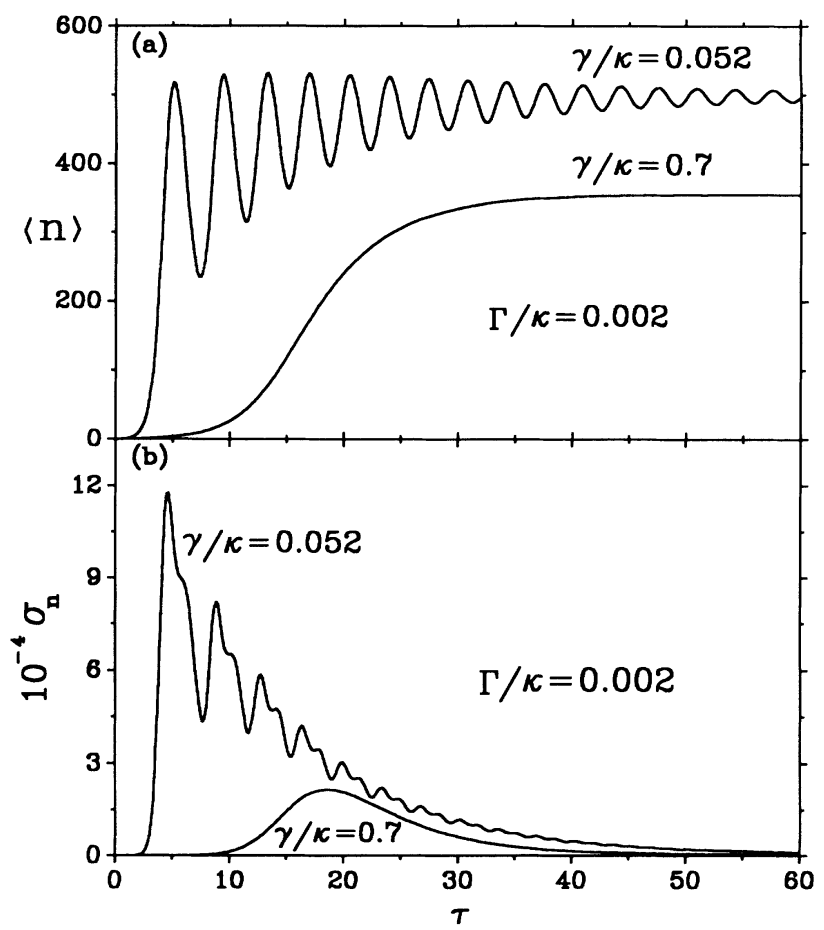

FIG. 8. Evolution in the oscillatory regime compared with the same in the overdamped regime for photon-number average (a) and the photon-number variance (b) (coherentvacuum initial state).

evolution. A value of 1.50 for the passage-time variances has been obtained in all cases. This means that, at least, there is no dispersion in the width of the first peak. Assuming that only PT dispersion occurs (i.e., all curves have exactly the same shape), it is not difficult to understand the double-peak structure if we interpret the dispersion in a variable as the projection of an oscillatory band of constant width (the PT variance) onto the axis corresponding to the considered variable. If the PT-probability distribution is symmetric with respect to the most likely trajectory, the position of the local mini-

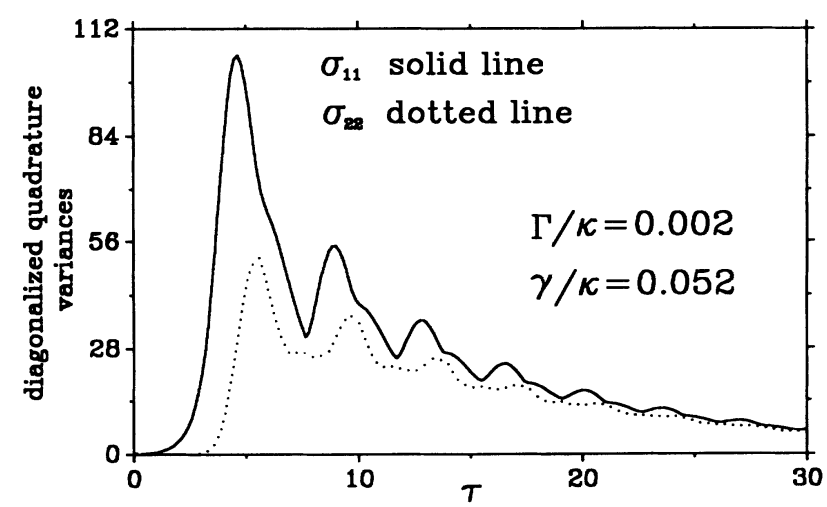

FIG. 9. Evolution of the quadrature variances in the oscillatory regime (coherent-vacuum initial state). 


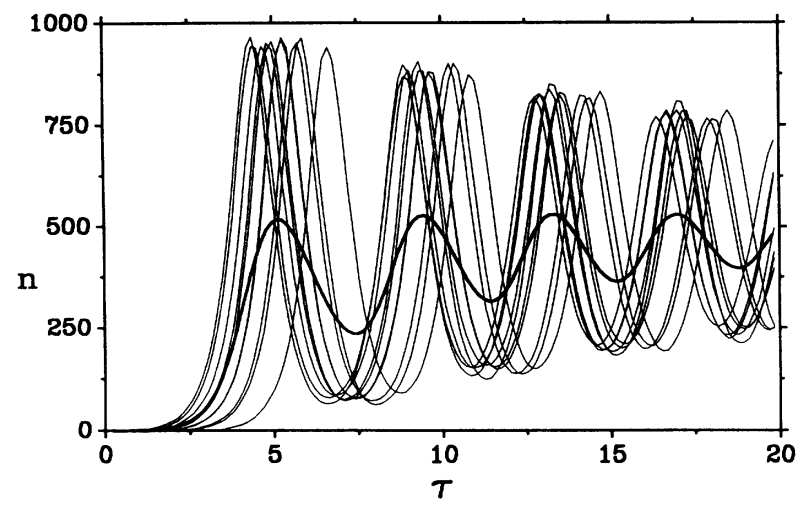

FIG. 10. Ten switching events for the photon number corresponding to the oscillatory case of Fig. 8(a). The thick line represents the average value.

mum in the variance will coincide with that of the local maximum of the average of the corresponding variable $[16,17]$. Since in our case the local maximum is located before the local minimum of the variance we conclude that the PT-probability distribution is not symmetric with respect to the most likely trajectory. In general this structure will appear in regions in which PT dispersion is dominant and roughly constant. This can occur, at least at the beginning of the evolution, if the starting times show large dispersion. Our model system has a two-dimensional real phase space and bistability with equal probabilities for both stationary states. Because of the two-dimensional and real character of our model, if there is an oscillatory regime it must have a conservative limit and both generalized position and momentum must oscillate around steady states. This implies that the starting directions in the unstable point cannot aim at the stationary points. Therefore the deterministic flow around the unstable point can produce a large dispersion in the starting times. This is not the case of the standard parametric amplifier [15] in which the larger dimensionality of the phase space allows the stationary states to

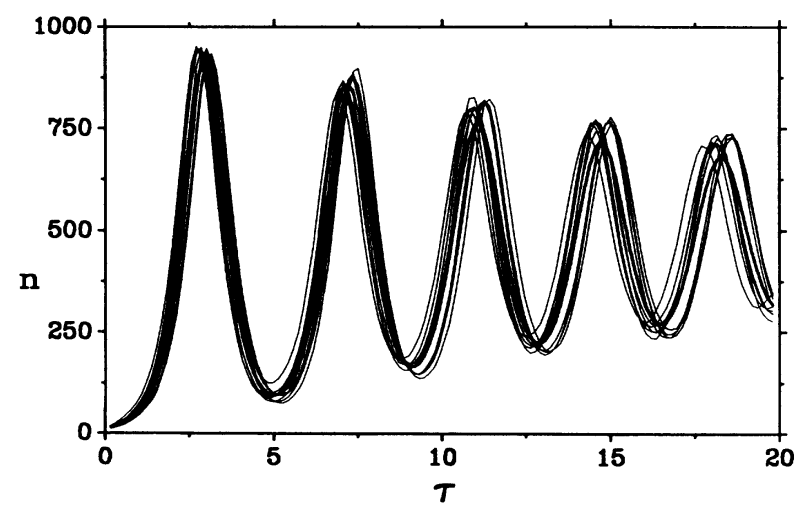

FIG. 11. Ten switching events for the photon number with the same parameter values as in Fig. 10 but with an initial coherent state such that $\langle x(0)\rangle=21$, and $\langle y(0)\rangle=-5.3$. The thick line represents the average value.

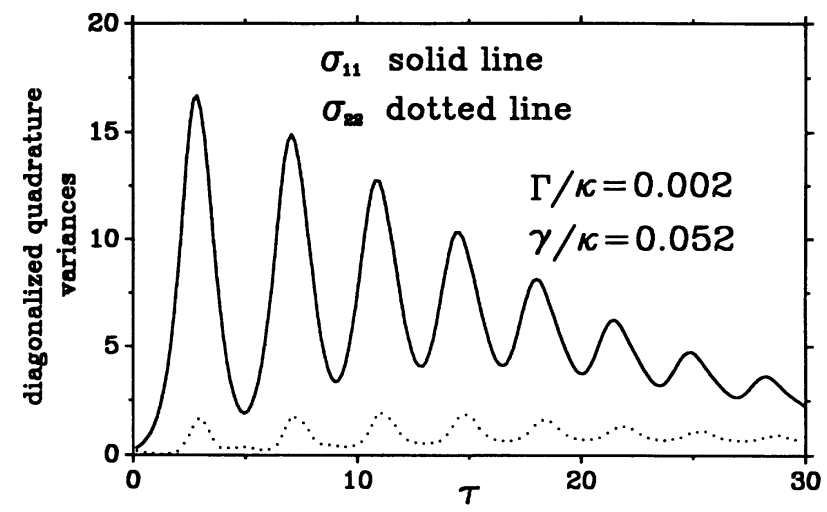

FIG. 12. Evolution of the quadrature variances for the case of Fig. 11.

settle onto the starting directions of the unstable state.

Figure 11 shows the same switching events for the photon number as Fig. 10 but with an initial state different from the vacuum. As can be clearly seen upon inspection of the figure, the input of a nonvacuum state results in a smaller dispersion of the initial times. At the same time a dispersion on the oscillation frequencies appears due to the influence of the fourth-order term at the initial time (self-phase-modulation $[4,5,18])$. The double structure in the variances now tends to disappear as is shown in Fig. 12.

It should also be noted that the oscillations occur around an exponential decay and not around a horizontal line as in the standard parametric amplifier. Again this is due to the noncoincidence of the starting directions with those which stationary states settle onto.

\section{ADEQUACY OF THE MAIN APPROXIMATION}

For a correct application of the semiclassical phasespace representation used herein it is necessary that the quantum fluctuations are appreciably smaller than the mean value. As we have computed statistical quantities over the whole evolution, an analysis of the region where the approximation holds must be done. Following Tombesi we use as a criterium for the validity of the approximation the smallness of the parameter

$$
S(\tau)=\left|\frac{\left(\alpha^{2}\right)_{\mathrm{cl}}-(\alpha)_{\mathrm{cl}}^{2}}{(\alpha)_{\mathrm{cl}}}\right|,
$$

which has the expression in quadrature variables

$$
S(\tau)=\left(\frac{\left(\sigma_{x}-\sigma_{y}\right)^{2}+4 \sigma_{x y}^{2}}{(x)_{\mathrm{cl}}^{2}+(y)_{\mathrm{cl}}^{2}}\right)^{1 / 2},
$$

where $\sigma_{x}, \sigma_{y}$, and $\sigma_{x y}$ are the components of the variance matrix at each $\tau$. Figure 13 shows $S(\tau)$ for several cases. Comparing the $S(\tau)$ curves with the corresponding counterparts for the variances it is observed that the approximation is not valid in the regions around the maximum of the variances as can be expected. Therefore the results should be taken as qualitative in these regions. 


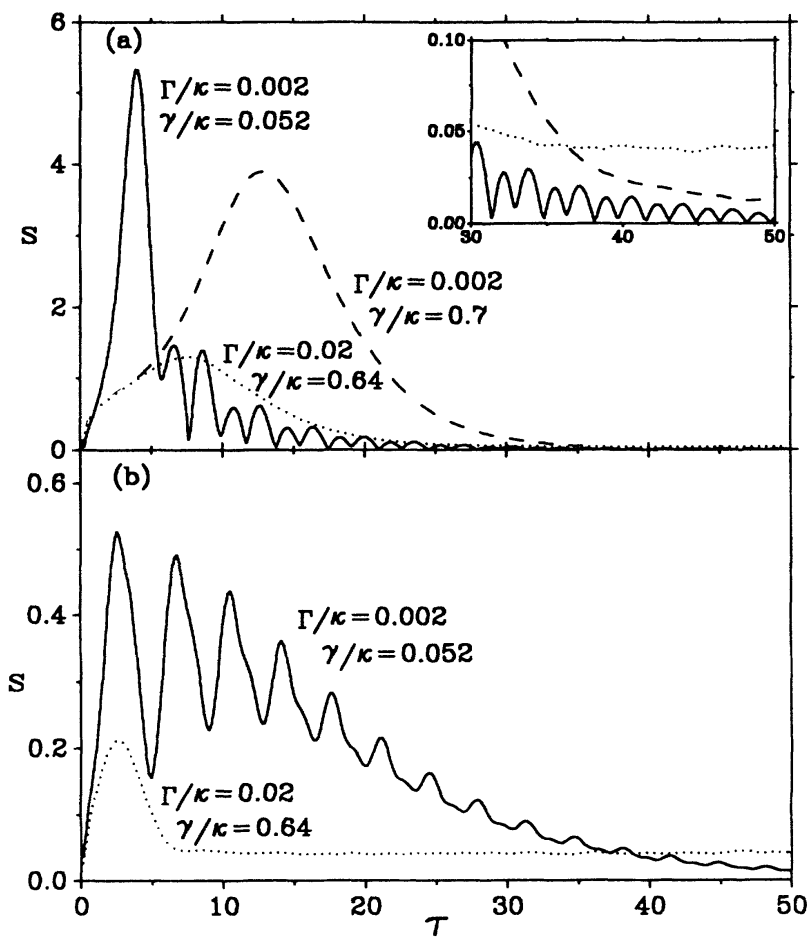

FIG. 13. Evolution of the parameter $S$ (variance-average relation) for a coherent-vacuum initial state (a) and for a nonvacuum initial state (b). The inset shows the evolution below the value $S=0.1$.

In cases where the initial state is the vacuum, the oversqueezing region does not present a small enough value of $S$. However, in this region there is no influence of the fourth-order term and the method is exact [6]. Setting $\Gamma / \kappa=0$ in the simulations, no discrepancy with the cases $\Gamma / \kappa \neq 0$ has been found in these regions within our numerical precision. The same holds for the simulations made below threshold. In the oscillatory case, $S(\tau)$ takes small enough values in regions in which the characteristic oscillatory behavior is observed. Therefore, we can conclude that this behavior is not a consequence of the approximation taken.

It should be noticed that we have not taken into account the depletion in the pump which is expected to be important near threshold (some region in the overdamped regime). In due time a complete study of the model system including depletion in the pump will be reported.

\section{SUMMARY AND CONCLUSIONS}

We have considered here the squeezing properties of a model device which includes two- and four-photon interactions as well as a realistic account of beam losses. Such a model exhibits bistable behavior above threshold leading to a steady regime which shows quadrature squeezing. A detailed analysis of the stationary statistics has evidenced that the attained states do not have minimum uncertainty relations and show super-Poissonian photon statistics.

The study of the transient behavior above threshold when the initial state is a coherent vacuum has evidenced that the fourth-order term does not exert any effect at the first stages of the evolution, and therefore the results are comparable to those attained by a conventional parametric amplifier [15]. At these first stages the squeezing is the maximum attainable with a parametric amplifier since it shows the same dependence with respect to the pump-loss relation as in the case below threshold. It has to be noticed that the model herein studied enables the possibility of controlling the duration of this initial "oversqueezing" region in a pump-independent way since it is governed not only by the pump-loss relation but also by the relative values between the pump and the Kerr-effect term. On the other hand, for the case of nonvacuum initial states, the present results confirm the numerical calculations carried out by Gerry and Rodrigues [2] for a lossless case regarding the disappearance of the "oversqueezing" effect before it attains a value corresponding to a two-photon interaction. An oscillatory behavior has been found for low-loss cases. In particular, the time evolution of the variances shows a characteristic doublepeak structure similar to those found in recent papers $[16,17]$ on semiconductor-laser gain switching. A close look at several sets of trajectories has shown that the appearance of such double structure is caused by the large temporal dispersion, at least when the initial state is a coherent vacuum. For cases where the trajectories depart from a nonzero coherent state, a noticeable frequency dispersion is readily seen. Such an effect is caused by the fourth-order term and can be considered to be an analogue to the observed self-phase-modulation exhibited in optical fibers [18] under circumstances where the Kerreffect term plays a dominant role.

\section{ACKNOWLEDGMENT}

This work was supported in part by Grant No. TIC90/80 (DGICYT, Spain).
[1] P. Tombesi, in Quantum Optics IV, Proceedings of the Fourth International Symposium, Hamilton, New Zealand, 1986, edited by J.D. Harvey and P.F. Walls (Springer-Verlag, Berlin, 1986).

[2] C.C. Gerry and S. Rodrigues, Phys. Rev. A 36, 5444 (1987).

[3] P. García-Fernandez, P. Colet, R. Toral, M. San Miguel, and F.J. Bermejo, Phys. Rev. A 43, 4923 (1991).

[4] C.C. Gerry, Phys. Lett. A 124, 237 (1987).

[5] R. Tanaś and S. Kelich, Quantum Opt. 2, 23 (1990).

[6] T.W. Marshall and E. Santos, Phys. Rev. A 41, 1582 (1990).

[7] P. D. Drummond, K. J. McNeil, and D. F. Walls, Opt. Acta 27, 321 (1980); 28, 221 (1981). 
[8] G. Milburn and D. F. Walls, Opt. Commun. 39, 401 (1981).

[9] H. J. Carmichael, G. J. Milburn, and D. F. Walls, J. Phys. A 17, 469 (1984).

[10] R. Loudon and P. L. Knight, J. Mod. Opt. 34, 709 (1987).

[11] C. W. Gardiner, Handbook of Stochastic Methods (Springer, Berlin, 1985), Chap. 4.

[12] J. M. Sancho, M. San Miguel, S.L. Katz, and J. D. Gunton, Phys. Rev. A 26, 1589 (1982).

[13] M. Kalos and P. Whitlock, Monte-Carlo Methods. Volume 1: Basics (Wiley, New York, 1986).
[14] M. Wolinsky and H.J. Carmichel, Opt. Commun. 55, 138 (1985).

[15] H. J. Carmichael and M. Wolinsky in Quantum Optics IV (Ref. [1]).

[16] A. Mecozzi, P. Spano, and A. Sapia, Opt. Lett. 15, 1067 (1990).

[17] S. Balle, P. Colet, and S. San Miguel, Phys. Rev. A 43, 498 (1991).

[18] G. P. Agrawal, Nonlinear Fiber Optics (Academic, San Diego, 1989), Chap. 4. 